\title{
Chansons humanitaires, dépolitisation des conflits et moralisation des relations internationales à la fin de la Guerre froide ${ }^{1}$
}

La transformation de l'action humanitaire dans les dernières décennies du Xx ${ }^{e}$ siècle, après la guerre dite du Biafra (1967-1970), marque la fin du tiers-mondisme et accompagne la reconfiguration géopolitique survenue à la fin de la Guerre froide. Elle transforme également l'utilisation symbolique de certaines pratiques musicales en démocratie, la musique et les images (vidéoclips) étant désormais mobilisées au cœur des dispositifs de légitimation de l'action humanitaire grâce à l'action de médias de masse. En effet, certaines chansons font partie, de façon plus ou moins formelle, des campagnes de communication des organisations humanitaires à partir de la famine provoquée par la guerre civile éthiopienne entre 1983 et 1985, au moment où l'action humanitaire est montrée comme une preuve de la supposée supériorité morale du "monde libre" $"$.

Ce changement fonctionnel de la musique, traduit par l'essor des dispositifs musicaux humanitaires, s'inscrit dans un contexte précis de transformation politique, celui de la moralisation progressive des relations internationales. Comme l'affirme Rony Brauman, président de Médecins sans frontières (MSF), pendant ses activités en Éthiopie en 1984-1985, «l'humanitaire est une morale politique de substitution aux grands projets de transformation sociale, à la fin des utopies transformatrices et modernisatrices du $\mathrm{XX}^{\mathrm{e}}$ siècle $^{3}$ ". De fait, sur proposition de la Jordanie, l'Assemblée générale de l'ONU réfléchit depuis 1981 à la mise en place d'un "Nouvel ordre humanitaire international ", avec l'objectif d'" améliorer encore un

1. Cette recherche a bénéficié d'un soutien financier de l'École des hautes études en sciences sociales (EHESS) dans le cadre du projet de recherche "Création musicale, censure et politique symbolique dans les régimes autoritaires et totalitaires ".

2. Le concert organisé notamment par George Harrison en 1971 au Madison Square Garden de New York pour aider les victimes civiles du conflit armé au Bangladesh constitue un précèdent de cette transformation.

3. Entretien de l'auteur avec Rony Brauman, le 14 novembre 2012 à Paris. 
cadre international global tenant pleinement compte des instruments existants relatifs aux questions humanitaires ${ }^{4}$.

Bien que l'impact de l'action humanitaire dans les relations internationales et dans la géopolitique contemporaine soit analysé de façon exhaustive par des chercheurs issus de plusieurs disciplines des sciences humaines et sociales, le rôle de la musique dans la légitimation de ces actions et dans la construction d'imaginaires globalisés a fait l'objet de peu de travaux en langue française ${ }^{5}$. Dans cet article, je propose une analyse des principales caractéristiques des chansons humanitaires, que je considère comme faisant partie de dispositifs de politique symbolique plus larges, et du rôle de celles-ci dans le processus de moralisation des relations internationales à la fin de la Guerre froide ${ }^{6}$. Dans une première partie, j'analyserai donc les caractéristiques musicales, visuelles et discursives des dispositifs musicaux humanitaires afin de saisir leur mode de fonctionnement dans le contexte de la constitution d'un nouvel ordre humanitaire international. Ensuite, j'étudierai le rôle joué par ceux-ci dans la dépolitisation des conflits armés et de la gestion des catastrophes naturelles, ainsi que dans le déploiement d'une «politique de la pitié » et d'une « raison humanitaire " 7 .

Les exemples cités concernent principalement quatre dispositifs musicaux humanitaires et leur chanson respective : Band Aid (Do they know it's Christmas?), USA for Africa (We Are the World), Chanteurs sans frontières (Chanson pourl'Éthiopie) et enfin l'association Aznavour pourl'Arménie (Pour toi Arménie $)^{8}$. Si le choix de ces exemples n'est pas arbitraire, il est forcément sélectif et partiel, étant donné l'énorme quantité de dispositifs musicaux humanitaires qui ont produit des chansons dans des contextes extrêmement divers ${ }^{9}$. Ainsi, l'objectif de ce texte est-il de constituer le premier

4. Résolution 36/136 de l'Assemblée générale de l'ONU, 14 décembre 1981. Cette réflexion est prolongée en 1982 (résolution 37/201), puis est créée, en juillet 1983, une commission indépendante pour l'étude des questions humanitaires.

5. À quelques exceptions près, notamment l'article de Yasmine Carlet et Jean-Marie Seca, "Vingt ans de Live Aid : comment le charity rock a-t-il transformé l'engagement politique en musique populaire ?", Les cahiers psychologie politique, $\mathrm{n}^{\circ} 7$, juillet 2005. En ligne : http://lodel.irevues.inist.fr/ cahierspsychologiepolitique/index.php?id=1164.

6. J'ai défini auparavant les dispositifs de politique symbolique comme « un ensemble de stratégies hétérogènes, impliquant la production d'objets symboliques et leur mobilisation dans des relations de pouvoir, mise en œuvre par des acteurs et des institutions qui visent la légitimation ou la contestation d'un ordre social ». Luis Velasco Pufleau, "Autoritarisme, politique symbolique et création musicale. Stratégies de légitimation symbolique dans le Mexique postrévolutionnaire et le Nigeria postcolonial ", Revue internationale de politique comparée, 2012, vol. 19, nº 4, pp. 15-40.

7. Notions développées par Luc Boltanski, La Souffrance à distance, Paris, Gallimard, 2007 (1 $1^{\text {re }}$ édition : 1993), et par Didier Fassin, La Raison humanitaire. Une histoire morale du temps présent, Paris, Seuil/Gallimard, 2010.

8. A voir et entendre sur : www.youtube.com/watch?v=jdQWaOUq6c4; www.youtube.com/ watch? $=\mathrm{vWgRH} 3 \mathrm{~S} 0-8 \mathrm{Y}$; www.youtube.com/watch?v=-oLnNuO5N4A ; www.youtube.com/ watch?v=0SugqN9i82s

9. Pour ne citer que quelques-uns de ces dispositifs, des classiques aux plus récents en passant par certains plus étonnants : le collectif Fondation Québec-Afrique avec la chanson Les yeux de la faim en 1985 (http://www.youtube.com/watch?v=TDEcZppYv7A) ; Pharmaciens sans frontières avec la chanson Il faut le secourir en 1989 (http://www.youtube.com/watch?v=rgBqhGsBd5E) ; le collectif Croatian Band Aid (Hrvatski Band Aid) avec la chanson patriotique MY Homeland 
jalon d'une histoire musicale et politique de la chanson humanitaire de la fin de la Guerre froide jusqu'à nos jours.

\section{CARACTÉRISTIQUES DES DISPOSITIFS MUSICAUX HUMANITAIRES}

À partir de la fin de 1984, des musiciens et des personnes issues du monde de la télévision et du cinéma lancent, afin d'aider les victimes de la famine en Éthiopie, plusieurs initiatives médiatisées par les grandes chaînes de télévision nationales. Parmi les premiers, le chanteur Bob Geldof forme en Grande-Bretagne le collectif Band Aid et enregistre en novembre 1984 la chanson Do they know it's Christmas?, avec, entre autres, les chanteurs Bono, Phil Collins et Sting. Il organise en juillet 1985 le Live Aid, deux concerts simultanés à Londres (Wembley Stadium) et à Philadelphie (JFK Stadium), retransmis en direct par la radio et la télévision, et réunissant selon les organisateurs environ 1,5 milliard de spectateurs, essentiellement en Europe et en Amérique du Nord. Au total, entre 1985 et 1991, le projet draine au moins 144 millions de dollars, gérés par le Band Aid Charitable Trust ${ }^{10}$. Aux États Unis, des musiciens forment le collectif USA for Africa et enregistrent la chanson We Are the World, qui rencontre rapidement un grand succès. Parmi ces musiciens se trouvent Michael Jackson, Tina Turner, Lionel Ritchie, Ray Charles, Stevie Wonder et Bob Dylan; ils vendent environ 7 millions d'exemplaires et récoltent plus de 60 millions de dollars ${ }^{11}$.

En France, le chanteur Manu Dibango réunit des musiciens africains dès la fin de 1984 pour enregistrer leur Tam Tam pour l'Éthiopie. Puis, sous l'impulsion de Renaud et de Valérie Lagrange, se forme début 1985 l'association Chanteurs sans frontières dont l'objet est «l'aide aux victimes de la famine dans toute partie du monde, sans restriction, en dehors de toute considération quelconque d'ordre politique ou social, ceci dans un but exclusivement d'assistance et de bienfaisance ${ }^{12}$ ». Ils enregistrent la Chanson

(Moja domovina) durant le conflit armé en Ex-Yougoslavie, en 1991 (http://www.youtube.com/ watch $\left.\mathrm{v}_{\mathrm{v}}=\mathrm{p} 5 \mathrm{vhy} 0 \mathrm{RP} 8 \mathrm{aU}\right)$; le collectif Paris-Africa en France avec la chanson Des richochets en 2011 (www.unicef.fr/contenu/actualite-humanitaire-unicef/paris-africa-1-unisson-pour-les-enfants-2011-10-20) ; ou le collectif United Voices of Mali avec la chanson Mali-ko, dans le contexte du conflit armé malien en 2013 (www.youtube.com/watch? $=091 \mathrm{a} 5$ nqbAb8).

10. D’autres sources parlent de 225 millions de dollars (Bénédicte Rey, "Band Aid, une vague de solidarité inégalée qui a servi d' "impulsion" ", AFP Infos Mondiales, jeudi 26 novembre 2009). Toutefois, la somme exacte des fonds récoltés à ce jour est invérifiable, le Band Aid Charitable Trust donnant le chiffre de 144 millions pour la période 1985-1991 (http://www.live8live.com/docs/batwithlovefrombandaid\%20.pdf, consulté le 20 août 2013). Des détails complémentaires sur la répartition des sommes récoltées par les ventes des DVD et des CD du Band Aid et du Band Aid 20 sont donnés par la BBC (http://news.bbc.co.uk/2/hi/entertainment/4055325.stm, site consulté le 31 mai 2013).

11. http://www.usaforafrica.org/We_Are_the_World/introduction.html, site consulté le 21 août 2013.

12. "Statuts association "Chanteurs sans frontières" (Article 2. Objet) ", Sous-Préfecture de Boulogne-Billancourt, 12 juin 1985. 
pour l'Éthiopie, qui remporte un grand succès commercial et versent la quasitotalité des fonds récoltés - plus de 1,7 million d'exemplaires vendus ${ }^{13}$, environ 23 millions de francs (plus de 3 millions d'euros) - à MSF pour faire face à l'urgence humanitaire en Éthiopie.

Afin d'examiner la façon dont les dispositifs musicaux humanitaires légitiment et promeuvent l'action humanitaire, il est indispensable d'analyser leurs caractéristiques et leur mode de fonctionnement. Pour cela, je me fonderai sur les catégories d'analyse proposées par Roger Pouivet à propos de l'ontologie du rock ${ }^{14}$. Pour cet auteur, les œuvres rock sont des « artefacts-enregistrements ", c'est-à-dire des :

[...] œuvres musicales à instances multiples, sans cependant que cela dépende d'une notation (comme dans la musique savante écrite, quand la fidélité à la partition garantit l'identité de l'œuvre), mais grâce à un processus matériel de production (comme la gravure [la fabrication d'un enregistrement]) permettant leur diffusion généralisée ${ }^{15}$.

Ces " artefacts-enregistrements ", possédant des propriétés esthétiques, sont produits pour être économiquement accessibles au plus grand nombre - car " même si leur production peut être fort onéreuse, les produits sont eux-mêmes d'un prix modéré " - et pour être intellectuellement accessibles, car "ils ne supposent pas un degré élevé de culture humaniste ${ }^{16}$. Enfin, leur ontologie d'œuvres-enregistrements implique une disponibilité (de reproductibilité, d'accessibilité et de diffusion) permettant aux auditeurs de maitriser des émotions, de "les prendre en charge, soit pour les renforcer ou les atténuer, soit pour les modifier, plus ou moins radicalement ", en fonction de l'ensemble des propriétés fonctionnelles des œuvres ${ }^{17}$.

Cette définition de l'identité, des processus de création et de l'accessibilité des œuvres rock est particulièrement pertinente pour la chanson humanitaire. Les chansons humanitaires sont créées et mises en scène par les vidéoclips dans le studio d'enregistrement. Leur but étant de récolter rapidement une grande quantité de fonds, elles sont diffusées largement par les médias de masse afin de toucher le plus ample public et de vendre le plus grand nombre d'exemplaires possibles. L'œuvre est l'enregistrement (audio et vidéo) car elle sera donnée en concert à très peu d'occasions, lors de grandes manifestations ritualisées, contrairement à leur diffusion massive par la radio, la télévision ou Internet. D'ailleurs, les concerts humanitaires sont composés de répertoires propres à chaque musicien qui y participe, la chanson humanitaire constituant l'apogée

13. La Chanson pour l'Éthiopie est le deuxième "tube " le plus vendu des années 1980, juste derrière la Danse des canards ("Le top France des 80's ", Le Parisien, mercredi 11 janvier 2012).

14. Notamment dans "L'Ontologie du rock ", Rue Descartes, 2008, n 60, Paris, pp. 20-37, ainsi que dans Philosophie du rock, Paris, Puf, 2010.

15. Roger Pouivet, "L'Ontologie du rock ", op. cit., p. 23.

16. Idem.

17. Roger Pouivet, Philosophie du rock, op. cit., pp. 211-241 et p. 212 pour la citation. 
du rituel, le moment où la communauté (artistes et public) se rassemble pour aider les victimes, entonnant la même chanson.

Étant donné que les chansons humanitaires partagent des stratégies de création et de diffusion communes, ainsi que les mêmes codes musicaux et visuels (pochettes des disques et vidéoclips) et la même esthétisation des émotions, il est possible de les considérer comme un genre musical à part entière. D'ailleurs, les " artefacts-enregistrements » que sont ces chansons - et leurs vidéoclips - sont forcément accompagnés de discours (construction de figures de victimes), d'injonctions morales (nécessité de sauver les victimes) et de rituels médiatisés (concerts, apparitions des artistes dans des journaux télévisés, etc.). C'est l'ensemble de ces stratégies, discours, objets et rituels qui constitue le dispositif musical humanitaire. L'un des fondements de ces dispositifs est l'élaboration médiatique d'une fiction selon laquelle ils seraient spontanés, urgents, désintéressés et gratuits de la part des participants. Cette fiction est mise en scène par l'ensemble des discours des artistes et des médias, ainsi que par les textes des chansons et l'action des artistes montrée dans les vidéoclips ${ }^{18}$.

En effet, d'une façon générale, les chansons humanitaires partagent implicitement les mêmes fondements que l'action humanitaire : invocation de l'urgence, approche universaliste des victimes et exaltation de la vie. Parmi leurs principales caractéristiques musicales et discursives, nous pouvons souligner :

1. Leur ancrage dans une culture musicale - rock, variété, gospel partagée par la plus grande partie du public visé, par l'emprunt des codes, des formes et des structures musicales de ces répertoires. Concernant les formes musicales, il s'agit généralement des fonctions formelles des chansons populaires (articulation en couplets et refrains, avec la présence éventuelle d'un «pont " dans la section centrale du refrain), présentant une opposition mélodique et rythmique entre les parties et une progression dramatique vers la section finale (climax), la plupart du temps constituée par la répétition en crescendo du refrain pouvant être accompagnée de solos instrumentaux ${ }^{19}$.

2. La construction discursive des communautés morales : nous (donneurs) en opposition à eux (victimes) avec, éventuellement, un passage à une hypothétique communauté globale par l'identification ou l'unification des deux communautés (We Are the World). Les processus d'identification avec les victimes sont renforcés par la mobilisation d'émotions, telles la pitié ou la culpabilité : "Ce soir remercie Dieu que ce soient eux et pas toi " chante Bono $^{20}$, leader du groupe U2 et compagnon de Geldof dans

18. Pour un exemple : http://www.unicef.fr/contenu/actualite-humanitaire-unicef/paris-africalhistoire-dune-belle-mobilisation-pour-la-corne-de-lafrique-2011-11-02, site consulté le 26 août 2013.

19. Pour une analyse approfondie de ces formes, Franco Fabbri, "La chanson ", in Jean-Jacques Nattiez (dir.), Musiques. Une encyclopédie pour le XXr siècle, vol. 1, Paris, Actes Sud / Cité de la Musique, 2003, pp. 674-702 ; ainsi que Claude Abromont, Eugène de Montalembert, Guide des formes de la musique occidentale, Paris, Fayard / Henry Lemoine, 2010, pp. 40-43.

20. En version originale anglaise: "Tonight thank God it's them instead of you ". 
l'entreprise Band Aid, dans Do they know it's Christmas? ; "Loin du cœur et loin des yeux, de nos villes de nos banlieues, l'Éthiopie meurt peu à peu ", chantent en chœur les Chanteurs sans frontières dans leur Chanson pour l'Éthiopie.

3. La construction des figures des victimes - la « représentation humanitaire des victimes ", selon la catégorie de Philippe Mesnard ${ }^{21}$ - de façon globale et homogène, avec des caractéristiques, des souffrances et des besoins uniques et partagés. Elles ne sont pas définies dans leur profondeur sociologique, historique ou géopolitique. Ce déni d'individualité aux victimes est l'une des conditions qui permet leur universalisation et la dépolitisation de leur situation dans le discours véhiculé par les chansons humanitaires. En effet, " la politique de la pitié considère les malheureux assemblés en masses, même si elle doit [...] pour inspirer pitié, prélever dans cette masse des misères singulières ${ }^{22} "$.

4. La construction d'un ailleurs imaginé et anhistorique, généralement l'Afrique, où les victimes se trouveraient en attente de secours. Cette simplification géographique et/ou historique est exacerbée par le caractère urgent de l'aide - comme si approfondir la compréhension de la situation pouvait retarder l'arrivée de celle-ci et, de ce fait, provoquerait des morts évitables - contribuant à la dépolitisation des conflits armés et de la gestion des catastrophes naturelles.

Les chansons humanitaires sont accompagnées obligatoirement par des vidéoclips qui renforcent ou complètent les caractéristiques énumérées cidessus, dédoublant l'efficacité du rôle des chansons au sein des dispositifs. Ces images sont tournées dans les studios d'enregistrement ou sont issues la plupart du temps, en ce qui concerne les victimes, des journaux télévisées ou de reportages sur les zones sinistrées. Par le biais de l'intertextualité, les paroles et les images peuvent se renforcer mutuellement ou prendre de nouvelles significations. Parmi les principales caractéristiques des vidéoclips des chansons humanitaires il est possible de remarquer celles-ci :

1. La mise en scène des vidéoclips montrant les artistes en train d'enregistrer les chansons humanitaires dans le huis clos du studio, en contraste avec les victimes qui se trouveraient dans de grands espaces désolés. Cette construction est l'un des fondements du mythe de l'urgence et de la spontanéité des dispositifs car elle présuppose que les artistes ont à peine eu le temps de se réunir afin d'enregistrer la chanson. La mise en scène de l'urgence est symbolisée par l'utilisation des casques d'enregistrement Hi-Fi, par la lecture des paroles en direct (comme si les chanteurs n'avaient pas eu le temps d'apprendre le couplet ou le refrain de la chanson ${ }^{23}$ ) et par l'interaction avec les techniciens du studio.

21. Philippe Mesnard, La Victime écran. La représentation humanitaire en question, Paris, Textuel, 2002.

22. Luc Boltanski, La Souffrance à distance, Paris, Gallimard, 2007 (1 $1^{\text {re }}$ édition : 1993), p. 23.

23. J'utilise par convention lexicale le pluriel (masculin) " chanteurs ", mais les collectifs des chansons humanitaires comptent un grand nombre de chanteuses en leur sein. 
2. La mise en scène de la constitution et de l'émulation de la communauté morale durant la chanson. En effet, les chansons humanitaires étant chantées par un collectif d'artistes, la plupart du temps les vers des premiers couplets sont chantés en solo, puis en duo ou en trio pour enfin arriver au refrain chanté en chœur. Les vidéoclips mettent soigneusement en scène cette progression, ne montrant jamais la totalité des chanteurs dès les premiers couplets. Cette stratégie visuelle donne l'impression que les membres du collectif se regroupent peu à peu, qu'ils arrivent durant la chanson en crescendo jusqu'au climax final - constitué généralement par le refrain répété plusieurs fois en chœur, parfois accompagné par des solos "improvisés " de certains musiciens - où les chanteurs se comptent par dizaines.

3. L'absence des victimes dans les vidéoclips des premières chansons humanitaires, tout en instaurant un clivage symbolique extrêmement fort entre eux (victimes) et nous (donneurs), et ceci, au moins jusqu'en 1989 lors de la sortie de Pour toi Arménie. Néanmoins, les images des victimes sont omniprésentes sur les chaînes de télévision et parfois elles peuvent être accompagnées par les chansons humanitaires en fond sonore et sans commentaires, comme c'est le cas en France, à la sortie de la Chanson pour l'Éthiopie, au journal télévisé d'Antenne $2^{24}$. Au moment où les images des victimes sont intégrées dans les vidéoclips à la fin des années 1980, l'opposition entre les deux communautés est exacerbée et devient insoutenable : le spectateur ne peut alors que s'identifier avec les chanteurs ${ }^{25}$.

4. L'esthétisation des émotions des chanteurs lors des enregistrements. Les gestes, les postures et les expressions des chanteurs - lesquels se montrent séduisants, satisfaits de leur participation au projet et, dans de nombreux cas, s'amusant ou prenant un plaisir manifeste - constitue un des points communs entre les vidéoclips des chansons humanitaires. Cette satisfaction est précédée la plupart du temps par des moments de gravité (généralement durant les premiers couplets), où les chanteurs se montrent soucieux des problèmes pour lesquels ils se rassemblent dans le studio, avant de les transformer dans un espoir chanté en chœur dans les refrains : "Nourris le monde, fais-leur savoir que c'est Noël ", chantent les membres du Band Aid à l'apothéose de leur chanson ${ }^{26}$. L'identification des spectateurs avec les chanteurs passe donc par ces étapes de préoccupation et de culpabilité pour se transformer ensuite en jouissance par le don, par l'action de donner afin de venir en aide aux victimes : "Nous sommes ceux qui rendent les jours meilleurs, alors commençons à donner », entonnent en chœur les membres de USA for Africa ${ }^{27}$.

24. www.ina.fr/video/CAB86022011/chanson-francaise-ethiopie-video.html, site consulté le 3 juin 2013.

25. Pour un exemple saisissant de ce point $:$ http://www. unicef.fr/contenu/actualite-humanitaireunicef/paris-africa-decouvrez-le-clip-des-ricochets-2011-11-04, site consulté le 26 aout 2013.

26. La citation en version originale anglaise: "Feed the world, Let them know it's Christmas time ".

27. En version originale anglaise : "We are the ones who make a brighter day, so let's start giving". 
Les chansons humanitaires sont un excellent exemple du processus de transfert des codes, des pratiques et des objets musicaux dans des pays et des situations diverses. Effectivement, quel que soit le contexte géopolitique, toutes les chansons humanitaires montrent des hommes et des femmes avec des casques sur les oreilles, pressés par l'urgence humanitaire, chantant devant les micros d'un studio d'enregistrement afin de récolter des fonds pour secourir les victimes. Les discours médiatisés sont également les mêmes : devant l'insupportable souffrance des victimes, nous avons eu l'idée de faire une chanson pour eux, pour les enfants de... Ainsi, les chansons humanitaires peuvent-elles être considérées comme des hymnes médiatiques des démocraties libérales, destinés à fédérer des communautés morales aussi éphémères qu'impuissantes, au moment où l'humanitaire remplace les idéologies politiques et émancipatrices du $\mathrm{Xx}^{\mathrm{e}}$ siècle.

\section{NOUVEL ORDRE HUMANITAIRE INTERNATIONAL ET DÉPOLITISATION DES CONFLITS À LA FIN DE LA GUERRE FROIDE}

L'efficacité de ces dispositifs musicaux humanitaires repose sur la transformation de questions politiques en questions morales, cachant la profondeur historique et géopolitique des problèmes et rendant inattaquables les dispositifs humanitaires, puisqu'ils seraient moralement bons, nécessaires et urgents $^{28}$. La victimisation des populations africaines concernées par les discours humanitaires du Band Aid et des Chanteurs sans frontières, notamment, opère ainsi une transformation de la dimension politique du conflit armé éthiopien et des conséquences de l'action humanitaire en une question morale. Comme le montre Luc Boltanski, "le déploiement d'une politique de la pitié suppose donc deux classes d'hommes, inégaux, non sous le rapport du mérite, comme dans une problématique de la justice, mais uniquement sous celui du bonheur $"{ }^{29}$. Ainsi, Bob Geldof reçoit-il le prix de la Third World Foundation en 1986 pour son « immense croisade personnelle contre la souffrance et l'indifférence humaines, à laquelle des millions de personnes partout dans le monde se sont identifiées ${ }^{30}$ ". Pour la Foundation, il aurait " créé une communauté de personnes concernées dans le monde entier à partir d'une communauté d'artistes avec Band Aid. De spectateurs passifs, ils sont devenus des participants actifs, réunis autour du monde ${ }^{31} »$.

28. Parmi les premières critiques relatives aux implications morales et politiques de l'action humanitaire, voir notamment Bernard Hours, L'Idéologie humanitaire ou le spectacle de l'altérité perdue, Paris, L'Harmattan, 1998.

29. Luc Boltanski, op. cit., pp. 24-25.

30. En version originale anglaise : "Mammoth personal crusade against human suffering and human indifference, with which millions the world over identified ", "Third World Foundation News: 1986 Third World Prize ", Third World Quarterly, vol. 9, n² 2, 1987, p. 699.

31. En version originale anglaise : "He had created a world-wide community of concerned people. No longer passive spectators, they had become active participants ", "Third World Foundation News: 1987 Third World Lecture ", Third World Quarterly, vol. 9, n³, 1987, p. 1044. 
L'apolitisme supposé des acteurs humanitaires, véhiculé et renforcé par ces chansons, rend très difficile une critique politique frontale de ces dispositifs, d'autant plus qu'elle impliquerait un débat que l'urgence intrinsèque de leur action ne permet pas. Dans la plupart des discours médiatiques, les acteurs évacuent les questions diplomatiques et stratégiques de leur action, créant un semblant d'action fraternelle qui, dans de nombreux cas, nuit à la compréhension historique des conflits et rend difficile la mise en place de solutions politiques et économiques durables pour les populations affectées ${ }^{32}$. Rony Brauman parle ainsi de l'action humanitaire en Éthiopie en 1985 :

L'impression qu'on donnait malgré tout c'était qu'on était tous ensemble, unis, main dans la main : gouvernements étrangers, gouvernement éthiopien, Nations Unies, ONG pour lutter contre les conséquences de ce tremblement de terre climatique qu'avait été la sécheresse en Éthiopie et qu'avec notre bonne volonté, nos chansons et notre amour, on allait régler tout ça. Donc tout ça était très légitimiste. C'est un peu le problème et la limite du discours humanitaire qui ne peut pas être une politique de remplacement ${ }^{33}$.

Dans le fonctionnement de ces dispositifs, les catastrophes naturelles et les conflits armés produisent des "victimes " qu'il faut rendre visibles médiatiquement en mettant en scène le danger et l'urgence des besoins auxquels elles sont soumises. Valérie Lagrange, à l'origine, avec le chanteur Renaud, de la fondation de l'association Chanteurs sans frontières, justifie la poursuite de leur campagne en faveur des victimes éthiopiennes face aux critiques, minimisant les problèmes politiques et exacerbant l'identification de tout un chacun avec ces victimes dans une perspective moralisatrice. Ainsi, devant le constat des difficultés politiques et logistiques pour acheminer l'aide en Éthiopie, affirme-t-elle :

Si sur 10 millions de personnes, il y a 50000 qui sont sauvées, si je fais partie de ces 50 000, je suis bien contente d'en faire partie. Je pense qu'il faut donner, il faut continuer l'action, quoi qu'il arrive et malgré toutes les choses politiques qui s'interposent ; ce n'est pas grave, il faut donner et c'est tout ${ }^{34}$.

L'apparition des dispositifs musicaux humanitaires coïncide avec ce que Didier Fassin nomme le "gouvernement humanitaire ", c'est-à-dire "le déploiement des sentiments moraux dans les politiques contemporaines $»^{35}$. Et par sentiments moraux, on entend « les émotions qui nous portent vers les malheurs des autres et nous font souhaiter les corriger ${ }^{36}$ ». Ce changement

32. Parmi l'abondante bibliographie sur ce sujet, notamment Jean-Pierre Olivier de Sardan, Anthropologie et développement. Essai en socio-anthropologie du changement social, Paris, Karthala (APAD), 1995 ; Bernard Hours, op. cit. ; Laëtitia Atlani-Duhault et Laurent Vidal (dir.), Anthropologie de l'aide humanitaire et du développement, Paris, Armand Colin, 2009 ; Didier Fassin, op. cit., ainsi que Didier Fassin et Mariella Pandolfi (eds.), Contemporary States of Emergency : The Politics of Military and Humanitarian Interventions, New York, Zone Book, 2010.

33. Entretien de l'auteur avec Rony Brauman, le 14 novembre 2012 à Paris.

34. Propos de Valérie Lagrange au journal télévisé d'Antenne 2, le 15 juillet 1985 (http://www. ina.fr/art-et-culture/musique/video/CAB86011680/plateau-valerie-lagrange.fr.html).

35. Didier Fassin, op. cit., p. 8.

36. Ibid., p. 7. 
est engagé durant les années 1980 par l'inclusion dans l'espace politique d'une raison humanitaire. La musique et les médias de masse jouent un rôle central au sein du dispositif de légitimation de cette raison par sa capacité à mobiliser des émotions, à créer des imaginaires globalisés et à fédérer des communautés imaginaires. Ainsi, la victimisation opérée par les dispositifs musicaux humanitaires favorise-t-il et légitime-t-il l'action humanitaire en tant que réponse aux problèmes géopolitiques et postcoloniaux. La dimension politique est remplacée, finalement, par une obligation morale à agir.

Parallèlement à la mise en place de cette raison humanitaire, durant toute la décennie 1980, la réflexion sur le nouvel ordre humanitaire au sein de l'Assemblée générale de l'ONU se poursuit ${ }^{37}$. En France, la réflexion sur les conditions et les conséquences des interventions humanitaires est portée par le gouvernement Mitterrand et certaines ONG (notamment MSF et Médecins du monde), réfléchissant à la mise en place d'un « devoir d'ingérence $^{38} »$. En effet, la défense de l'ingérence humanitaire apparaît en France par l'extrapolation à l'échelle internationale du délit de non-assistance à personne en danger du droit français : "Le devoir d'ingérence est présenté comme un devoir d'assistance à un peuple en danger ${ }^{39}$. " La notion du devoir d'ingérence est développée lors du colloque Droit et morale humanitaire, organisé du 26 au 28 janvier 1987 à Paris par Bernard Kouchner, lui-même identifié comme un French Doctor ${ }^{40}$, et le juriste Mario Bettati. François Mitterrand et son Premier ministre, Jacques Chirac, y sont présents.

En juin 1988, Kouchner est nommé par Mitterrand secrétaire d'État chargé de l'Action humanitaire. Il nomme alors son ami Bettati chargé de mission. Ils préparent alors un projet de résolution sur le droit d'assistance humanitaire, qui est défendu par Mitterrand lui-même le 29 septembre 1988 à New York devant l'Assemblée générale de l'ONU. Finalement, le 8 décembre 1988 est adoptée la résolution 43/131, intitulée "Assistance humanitaire aux victimes des catastrophes naturelles et situations d'urgence du même ordre ", invitant tous les Etats qui ont besoin d'une assistance humanitaire à collaborer à leur mise en œuvre en facilitant l'accès aux victimes. L'Assemblée de l'ONU se dit :

[...] profondément préoccupée par les souffrances des victimes de catastrophes naturelles et situations d'urgence du même ordre, par les pertes en vies humaines, les destructions des biens et les déplacements massifs de populations qui en résultent ${ }^{41}$.

37. En 1983, avec la résolution 38/125; en 1985, avec la résolution 40/126 et, en 1987, avec la résolution 42/120.

38. Pour une généalogie du passage du "droit " d'ingérence au " devoir »: Mariella Pandolfi et Alice Corbet, "De l'humanitaire imparfait ", Ethnologie française, 2011, vol. 41, pp. 465-472.

39. Jean-Baptiste Jeangène Vilmer, La Guerre au nom de l'humanité. Tuer ou laisser mourir, Paris, Puf, 2012, p. 156.

40. Bernard Kouchner est l'un des membres fondateurs de MSF en 1971 et puis de Médecins du monde (MDM) en 1979. L'une des images médiatiques les plus représentatives du French Doctor le montre portant un sac de riz sur l'épaule en 1992, alors qu'il est ministre de la Santé en France, dans le contexte de la famine provoqué par le conflit armé en Somalie, http://www.ina.fr/video/ CAB92069822/somalie-aide-humanitaire-video.html, site consulté le 3 septembre 2013.

41. Résolution 43/131 de l'Assemblée générale de l'ONU, 8 décembre 1988. 
Tout en réaffirmant la souveraineté, l'intégrité territoriale et l'unité nationale des États, la résolution considère que « le fait de laisser les victimes de catastrophes naturelles et situations d'urgence du même ordre sans assistance humanitaire représente une menace à la vie humaine et une atteinte à la dignité de l'homme ${ }^{42}$ ». De la même manière, la résolution souligne "l'importante contribution à l'assistance humanitaire qu'apportent les organisations intergouvernementales et non gouvernementales agissant dans un but strictement humanitaire ${ }^{43}$ ".

La veille de l'adoption de cette résolution, le 7 décembre 1988, deux séismes de magnitude 6.9 et 5.8 sur l'échelle de Richter secouent l'Arménie, faisant environ 55000 morts $^{44}$. À partir du 9 décembre, le pouvoir soviétique laisse entrer sans visa dans ses frontières, pour la première fois depuis la Seconde Guerre mondiale, des équipes de secours occidentales, civiles et militaires ${ }^{45}$. Un mois plus tard, Charles Aznavour organise l'enregistrement à Paris d'une nouvelle chanson humanitaire Pour toi Arménie. Plus de deux millions de disques sont vendus dans les semaines suivant sa sortie, les fonds récoltés étant versés à l'association Aznavour pour l'Arménie pour l'aide d'urgence aux victimes. Pour la première fois en France, les images de victimes figurent à l'intérieur du vidéoclip, aux côtés des chanteurs et des personnalités des médias, exacerbant la contradiction entre les communautés représentées afin de provoquer de la pitié envers les malheureux ${ }^{46}$. Le répertoire lexical en relation avec l'espoir est particulièrement mobilisé dans cette chanson - « Tes printemps fleuriront encore, tes beaux jours renaîtront encore " - mais aussi celui du religieux - "Après l'horreur, après la peur, Dieu soignera ton sol meurtri »- et de l'obligation morale à se relever - "Et même si tu maudis ton sort, dans tes yeux je veux voir, Arménie, une lueur d'espoir ». Les larmes d'Aznavour constituent le paroxysme émotionnel de la chanson, alors qu'il énonce le nom de sa patrie d'origine, l'Arménie, dans sa langue maternelle: Hayastan.

\section{CONCLUSION}

Les chansons humanitaires ont marqué les imaginaires de plusieurs générations, avec leurs cortèges de victimes, leurs médecins héroïques et leurs chanteurs bienfaisants. Pourtant, des années 1980 à nos jours, de nombreuses voix s'élèvent contre la réduction des populations au statut

42. Idem.

43. Idem.

44. http://perspective.usherbrooke.ca/bilan/servlet/BMEve?codeEve=1066, site consulté le 3 août 2013 .

45. http://www.ina.fr/video/CAB88047315/depart-pompiers-pour-armenie-video.html, site consulté le 19 août 2013.

46. https://www.youtube.com/watch?v=0XAsYugjBgE, site consulté le 29 août 2013. 
de victimes privées de toute individualité et de capacité à agir, telle la poétesse sud-africaine Khadija Tracey Heeger, qui résume de manière exemplaire ce refus dans l'introduction de son poème Étincelles d'Afrique (Cheche La Afrika) :

Je ne suis pas que famine et ruines, craie croulante, bâtiments noirs et dévastés.

Je n'existe pas pour qu'on me pleure.

Je ne suis pas un cortège de malheurs du

colonialisme, de l'apartheid, du féminicide, du génocide, de la xénophobie.

Mon sang est plus fort que mes chagrins.

Mon sang est plus épais que l'encre des livres d'histoire

qui dilapident ma vérité

et couvrent les tables de mes enfants de mensonges sur eux-mêmes.

Mes yeux sont ouverts.

J'ai cherché dans les feux et dans les flammes du temps

les joyaux de l'Afrique

enfin révélés ${ }^{47}$.

Cependant, l'utilité politique des dispositifs musicaux humanitaires est indéniable pour la légitimation des gouvernements des démocraties libérales, pour l'instauration et le maintien d'un certain ordre social à la fin de la Guerre froide. Ainsi l'affirme François Mitterrand, alors président de la République, lors de sa participation, le dimanche 28 avril 1985, à l'émission de TF 1 Ça nous intéresse, monsieur le Président. À cette occasion, le présentateur Yves Mourousi l'interroge, entre autres sujets soigneusement présélectionnés par l'équipe de communication du Président, sur l'action d'un certain nombre de chanteurs afin de récolter des fonds pour lutter contre la famine en Éthiopie :

Yves Mourousi : Chanter pour l'Afrique, chanter pour l'Éthiopie, cela sert à quelque chose d'après vous qui connaissez les organisations internationales ? Quand Renaud et tout un groupe de chanteurs français, quand les Américains chantent pour l'Éthiopie, vous avez l'impression que cela sert à quelque chose?

François Mitterrand : Oui, parce que cela crée l'émotion. Toute société fonctionne avec de l'émotion. Les sociétés ne sont pas que des mécaniques, et les gouvernements et les institutions ne sont pas des machines. L'imagination, la pitié, la solidarité, l'amour, cela existe, et si ces chanteurs chantent l'amour et, par là même, la main tendue pour secourir, ils font une œuvre utile, croyez-moi ${ }^{48}$.

47. En version originale anglaise : "I am not just famine and war torn, I am not just derelict white calcium and ravaged black edifices. I am not here to be the subject of mourning and a pageant for the ills of colonialism, apartheid, femicide, genocide, xenophobia. My blood is thicker than my sorrows. My blood is thicker than the ink in those history books that would squander the truth about me and deck the tables of my children with lies about themselves. My eyes are open. I have looked into the fires and in the flames of time. The jewels of Africa are revealed. " Khadija Tracey Heeger, Cheche La Afrika, reproduit dans le programme du Cape Cultural Collective présenté les 8 et 9 octobre 2013 à la Maison de la Poésie de Paris dans le cadre de la $42^{\mathrm{e}}$ édition du Festival d'Automne.

48. Intervention de François Mitterrand, président de la République, à l'émission de TF 1 Ça nous intéresse, monsieur le Président, Paris, Maison de la Radio, dimanche 28 avril 1985 (http://discours. vie-publique.fr/notices/857006300.html, site consulté le 27 mai 2013). 
Mitterrand soulève avec justesse le point fondamental du fonctionnement des dispositifs musicaux humanitaires, qui est constitué par leur charge émotionnelle, insistant sur l'importance des émotions dans le fonctionnement de la Cité, et de ce fait dans la légitimation du pouvoir. Les chansons humanitaires ont largement contribué à la maîtrise des émotions et au déploiement de la politique de la pitié dans l'espace public. Avec l'effondrement des régimes communistes à l'Est, l'humanitaire devient alors une nouvelle utopie du changement social, un moyen d'action afin de transformer le monde ici et maintenant qui serait hypothétiquement affranchi du poids des «idéologies ». Les chansons humanitaires prêchent l'aide aux malheureux sans pour autant chercher des responsabilités dans les conflits ou dans la gestion des crises. Comme le souligne Christophe Pirenne à propos du Live Aid, contrairement aux festivals de rock des années 1960 " il n'était plus question de mettre en cause le mode de fonctionnement des sociétés occidentales, mais de soigner les plaies de l'époque en adhérant au principe capitaliste de la guérison par l'argent ${ }^{49}$ ".

Les chanteurs engagés dans les dispositifs musicaux humanitaires font une " œuvre utile » aussi bien pour eux-mêmes et leurs carrières - le gain du capital symbolique par le fait de leur participation étant extrêmement profitable - que pour le maintien d'un certain ordre social et pour l'instauration du nouvel ordre humanitaire international. La mise en place de ce nouvel ordre s'intensifie en effet après la chute du mur de Berlin, notamment avec la résolution 45/100 (14 décembre 1990) de l'Assemblée générale, puis avec la résolution 668 (le 5 avril 1991) du Conseil de Sécurité de l'ONU concernant les réfugiés kurdes en Irak. L'année suivante il sera question du conflit armé en Somalie avec les résolutions 733 (23 janvier 1992), 794 (2 décembre 1992) et 814 (6 mars 1993) du Conseil de Sécurité, lesquelles constitueraient, pour Mario Bettati, "la consécration la plus vigoureuse du droit d'ingérence $^{50}$ ». En effet, toujours selon le juriste, durant les années 1990,

[...] le Conseil de sécurité, libéré des pesanteurs de la Guerre froide et dynamisé par l'essor du concept de " droit d'ingérence " développe, souvent à l'initiative de la France, une conception extensive de la notion de "menace contre la paix ». Il affirme notamment qu'une violation massive et grave des droits de l'homme dans l'ordre interne constitue une telle menace ${ }^{51}$.

Pour nombre d'acteurs politiques, le déploiement du concept, plus qu'ambigu, d' "ingérence humanitaire " est une avancée incontestable dans le progrès d'une certaine " conscience universelle ". En effet, à l'occasion de l'attribution du Prix Nobel de la paix à MSF en 1999, le président Jacques Chirac affirme que :

[...] c'est un hommage rendu à des hommes et à des femmes exceptionnels, qui dans des circonstances souvent dramatiques, souvent au péril de leur vie,

49. Christophe Pirenne, Une histoire musicale du rock, Paris, Fayard, 2011, p. 442.

50. Mario Bettati, «L'usage de la force par l'ONU », Pouvoirs, 2004, n 109, p. 112.

51. Idem. 
apportent aide et soutien de tout leur cœur à celles et ceux qui souffrent, soit de catastrophes humanitaires, soit de conflits, souvent d'ailleurs d'un autre temps et d'un autre âge. Il est heureux que ces gens qui incarnent en quelque sorte un progrès de la conscience universelle soient aujourd'hui récompensés ${ }^{52}$.

De même, son Premier ministre Lionel Jospin considère que les ONG « ont joué un rôle extrêmement important dans la progression des idées d'ingérence humanitaire, et donc ont fait progresser le droit international dans la pratique ${ }^{53}$ ». Néanmoins, cette hypothétique consécration du mythe français du droit d'ingérence peut être considérée comme le franchissement d'une «étape dans l'entreprise de moralisation du droit international public et des relations internationale ${ }^{54}$ ». Comme l'analyse Jean-Baptiste Jeangène Vilmer, l'essor des interventions humanitaires à la fin du $\mathrm{xx}^{\mathrm{e}}$ siècle s'explique aussi de façon négative : "L'intervention non humanitaire est de moins en moins possible, donc la seule manière d'intervenir est de le faire au nom de prétextes humanitaires, qui auparavant n'étaient pas requis ${ }^{55}$. " La politique de la pitié légitimée, elle, remplace la politique de la justice car il ne s'agirait plus de chercher des responsabilités politiques ou une justice sociale durable mais uniquement de faire cesser la souffrance. Comme l'explique avec justesse Luc Boltanski :

Pour une politique de la pitié, l'urgence de l'action à mener pour faire cesser les souffrances invoquées l'emporte toujours sur la considération de la justice. Depuis une telle perspective, c'est seulement dans un monde d'où la souffrance aurait été bannie que la justice pourrait faire valoir ses droits ${ }^{56}$.

Cependant, à partir de 2005, certains des dispositifs humanitaires classiques transforment significativement leurs discours, leurs stratégies et leurs objectifs. Fondés, organisés et dirigés notamment par Bob Geldof et Bono, le Live 8 et la campagne Make Poverty History ainsi que les ONG DATA (Debt, AIDS, Trade, Africa ${ }^{57}$ ) et $\mathrm{ONE}^{58}$, prêchent pour une lutte contre la pauvreté par la promotion d'une justice globale de nature abstraite avec des "solutions » économiques néolibérales. Les dirigeants du G8 collaborent fièrement avec ces mouvements, faisant des promesses que Geldof et Bono ne manquent pas de relayer pour demander aux dirigeants, quelques années plus tard, de les tenir ou d'en faire de nouvelles. Dans un prochain article, j'analyserai le rôle de la musique au sein de ces dispositifs, considérés par Lilie Chouliaraki comme post-humanitaires puisqu'ils ne visent plus à secourir les victimes d'une situation spécifique, mais favorisent des

52. http://www.ina.fr/video/CAB99041917/sonore-chirac-jospin-reaction-nobel-video.html, site consulté le 2 septembre 2013.

53. Idem.

54. Notamment Jean-Baptiste Jeangène Vilmer, op. cit., pp. 155-163. Pour la citation : Philippe Breton, "Ingérence humanitaire et souveraineté ", Pouvoirs, 1993, n 67, p. 61

55. Jean-Baptiste Jeangène Vilmer, op. cit., p. 133.

56. Luc Boltanski, op. cit., p. 24.

57. «Dette, sida, commerce, Afrique ", http://data.d202.org, site consulté le 3 septembre 2013.

58. http://www.one.org/fr, site consulté le 3 septembre 2013. 
engagements courts et de basse intensité fondés sur les pratiques de consommation des sociétés postindustrielles ${ }^{59}$. Il s'ensuit dans certains cas une récupération et une réappropriation ironique d'un vaste répertoire de chansons " engagées ", comme le montre la campagne En scène citoyens de ONE, afin de "contribuer à mettre fin à l'extrême pauvreté d'ici 2030 " ${ }^{60}$. Vaste projet.

Luis Velasco Pufleau

EHESS, Paris / Université de Salzbourg

59. Lilie Chouliaraki, «Post-humanitarianism: Humanitarian communication beyond a politics of pity ", International Journal of Cultural Studies, vol. 13, n² 2, 2010, pp. 107-126.

60. http://www.one.org/chansonsengagees, site consulté le 3 septembre 2013. http://www.one. org/chansonsengagees, site consulté le 3 septembre 2013. 